\title{
PERFIL NACIONAL DA ATENÇÃO FARMACÊUTICA PARA PACIENTES COM DOENÇAS CRÔNICAS NÃO TRANSMISSÍVEIS: UMA REVISÃO INTEGRATIVA
}

\begin{abstract}
NATIONAL PROFILE OF PHARMACEUTICAL CARE FOR PATIENTS WITH CHRONIC NON-TRANSMITTED DISEASES: AN INTEGRATIVE REVIEW
\end{abstract}

\author{
Odaisy Maria de Sousa ${ }^{1}$ \\ Beatriz Vitor do Nascimento ${ }^{2}$ \\ Maria Aparecida Mateus ${ }^{3}$ \\ Esther Gomes Pedrosa ${ }^{4}$ \\ José Guilherme Ferreira Marques Galvão ${ }^{5}$
}

\begin{abstract}
RESUMO: O objetivo deste estudo é enfatizar a importância da atenção farmacêutica aos pacientes com Doenças Crônicas não Transmissíveis e os fatores que contribuem para o desenvolvimento dessas doenças. Método: foi feita uma revisão integrativa do papel do farmacêutico sobre o impacto da atenção farmacêutica no manejo das Doenças Crônicas não Transmissíveis, realizada buscas nas bases de dados SciELO e no PubMed/MEDLINE. Selecionaram-se os termos-chaves, utilizando os descritores em ciências da saúde (DeCS): "pharmaceutical care" or "pharmaceutical services" and "chronic diseases" and "diabetes" and "hypertension" no SciELO e no PubMed/MEDLINE. Resultados: Segundo dados da organização mundial de saúde, em 2015 a hipertensão atingiu cerca de 1,13 milhões de pessoas no mundo, o diabetes atingiu 422 milhões de pessoas em 2014, e aproximadamente 23 milhões de pessoas no mundo tem diagnostico de insuficiência cardíaca congestiva. $O$ profissional farmacêutico tem papel importante na vida dos pacientes com Doenças Crônicas não Transmissíveis, atuando nos cuidados relacionados a esquemas posológicos, traçando estratégias para auxiliar no tratamento de pacientes com um nível inferior de escolaridade e informações (sobre sua condição ou tratamento que irá se submeter), inseguranças do paciente quando trata-se do medicamento que deverá ser utilizado, evitando

\footnotetext{
${ }^{1}$ Discente do curso de Farmácia da Faculdade Santa Maria - FSM.

2 Discente do curso de Farmácia da Faculdade Santa Maria - FSM.

${ }^{3}$ Discente do curso de Farmácia da Faculdade Santa Maria - FSM.

${ }^{4}$ Discente do curso de Farmácia da Faculdade São Francisco da Paraíba - FASP.

${ }^{5}$ Graduado em Farmácia pela Universidade Federal da Paraíba, mestre em Ciências Fisiológicas e professor dos cursos de Farmácia e Medicina da Faculdade Santa Maria - FSM.
} 
assim problemas relacionados aos medicamentos (PRM's), falta de adesão ao tratamento, redução de custos, redução na mobimortalidade, entre outros. Segundo os estudos lidos, os pacientes que foram atendidos por equipes que tinham farmacêuticos presentes, obtiveram melhores resultados, assim como maior adesão ao tratamento. Conclusão: $O$ presente estudo, nos leva a refletir sobre a prática clínica dos profissionais farmacêuticos na atenção básica aos pacientes de Doenças Crônicas não Transmissíveis, trazendo para os mesmos uma maior expectativa de vida, pelo fato de seguirem o plano farmacoterapêutico, graças a ajuda do farmacêutico, qualidade de vida, já que geralmente para muitas pessoas é um incomodo a pratica de medicar-se diariamente, dentre outros benefícios, como por exemplo, a redução de gastos.

Descritores: Atenção farmacêutica. Doenças crônicas. Diabetes. Hipertensão. Epidemiologia.

ABSTRACT: The aim of this study is to emphasize the importance of pharmaceutical care to patients with Chronic Noncommunicable Diseases and the factors that contribute to the development of these diseases. Method: an integrative review of the pharmacist's role on the impact of pharmaceutical care on the management of Chronic Noncommunicable Diseases was carried out, searches were carried out in the SciELO databases and in PubMed / MEDLINE. The key terms were selected using the descriptors in health sciences (DeCS): "pharmaceutical care" or "pharmaceutical services" and "chronic diseases" and "diabetes" and "hypertension" in SciELO and PubMed / MEDLINE. Results: According to data from the world health organization, in 2015 hypertension reached around 1.13 million people worldwide, diabetes reached 422 million people in 2014, and approximately 23 million people worldwide are diagnosed with congestive heart failure. The pharmaceutical professional has an important role in the life of patients with Chronic Noncommunicable Diseases, acting in the care related to dosage regimens, outlining strategies to assist in the treatment of patients with a lower level of education and information (about their condition or treatment that they will undergo), patient's insecurities when it comes to the medication that should be used, thus avoiding Problems Related to Medicines (PRM's), lack of adherence to treatment, cost reduction, reduction in mobility and mortality, among others. According to the studies read, patients who were treated by teams that had pharmacists present, obtained better results, as well as greater adherence to treatment. Conclusion: This study leads us to reflect on the clinical practice of pharmaceutical professionals in primary care for patients with Chronic Noncommunicable Diseases, bringing them a longer life expectancy, due to the fact that they follow the pharmacotherapeutic plan, thanks to the help of the pharmacist, quality of life, since it is usually a nuisance for many people to practice daily medication, among other benefits, such as, for example, reduced expenses.

Keywords: Pharmaceutical care. Chronic diseases. Diabetes. Hypertension. Epidemiology. 


\section{INTRODUÇÃO}

A profissão farmacêutica era considerada pela sociedade, no início do século $X X$, a referência quando o assunto era medicamento. Logo, entendia-se que o farmacêutico atuava exercendo influência sobre todas as fases do ciclo do medicamento. Ainda neste período, além da posse e dispensação do medicamento, a farmácia como profissão, acabava sendo responsável pela produção e manipulação de todo o arsenal, disponível á população, daquela época. (GOUVEIA, 1999).

O farmacêutico pode atuar no planejamento de estratégias em saúde visando promover um melhoramento na qualidade de vida dos pacientes. A interação entre farmacêutico e paciente faz com que os mesmos se sintam mais seguros em relação aos medicamentos que fazem uso. Desta forma, esses usuários acabam se sentindo mais à vontade para relatar algum evento adverso em consultas farmacêuticas. Paralelamente, o processo de atenção farmacêutica está ligado diretamente com o desenvolvimento de políticas públicas na tentativa de reduzir os índices de Doenças Crônicas Não Transmissíveis (DCNT), ao visar uma proposta constante de novas estratégias de tratamento que sejam condizentes com a realidade de seus pacientes.

As DCNT revelam crescentes índices de acometimentos incapacitantes e óbitos na população mundial. Cerca de $70 \%$ dos óbitos no mundo são decorrentes de comprometimentos oriundos dessa classe de patologias crônicas (LOPES, 2020). Quatro grupos de doenças - cardiovasculares, câncer, doença respiratória crônica e diabetes - responderam pela grande maioria desses óbitos (DUNCAN, 2012).

Segundo dados publicados pela Organização Mundial da Saúde (OMS), a hipertensão atingiu cerca de 1,13 bilhão de pessoas no mundo em 2015, e o diabetes atingiu 422 milhões de pessoas em 2014 (8,5\%). No Brasil, dados do Ministério da Saúde de 2016 mostraram que a hipertensão arterial sistêmica (HAS) aumentou $14,2 \%$ na última década e já atinge $25,7 \%$ da população brasileira com 18 
anos ou mais. Em relação ao diabetes mellitus (DM), 8,9\% da população referiu essa doença em 2016 (FAGUNDES, 2017).

Adicionalmente, a Insuficiência cardíaca congestiva (ICC) se apresenta como um problema para a saúde pública, devido à alta taxa de morbidade e mortalidade, gastos com medicação e internações principalmente para pacientes acima de 65 anos (SILVA, 2019). Assim, ao se estabelecer a partir de anormalidades cardíacas estruturais e/ou funcionais em decorrência da idade, a ICC comumente é entendida como uma doença mais prevalente em idosos.

Aproximadamente 23 milhões de pessoas são portadoras de ICC e 2 milhões de novos casos são diagnosticados a cada ano no mundo. No Brasil, estima-se que 6,4 milhões de brasileiros sofram de ICC, pode ocorrer de dois tipos: insuficiência cardíaca sistólica, que é quando o músculo cardíaco não consegue ejetar o sangue para fora do coração adequadamente e insuficiência cardíaca diastólica, que ocorre quando os músculos do coração ficam rígidos e não enchem de sangue facilmente (CAMPELO, 2018).

Desta forma, levando-se em conta que o impacto socioeconômico das DCNT vem ameaçando o progresso das Metas de Desenvolvimento do Milênio, incluindo a redução da pobreza, a equidade, a estabilidade econômica e a segurança humana, podendo atuar também como um freio no próprio desenvolvimento econômico das nações (DUNCAN, 2012), este presente trabalho tem como objetivo identificar através de uma revisão integrativa da literatura, a real eficácia da coparticipação do farmacêutico, na promoção a saúde em pacientes crônicos que possuam acesso aos processos de cuidados farmacêuticos. 


\section{METODOLOGIA}

\section{Estratégia de busca}

Inicialmente, uma revisão integrativa do papel do farmacêutico sobre o impacto da atenção farmacêutica no manejo das DCNT, foi utilizada como referência para inclusão de alguns estudos. Foram realizadas buscas nas bases de dados SciELO e no PubMed/MEDLINE. Ambos os sites foram acessados entre novembro e dezembro de 2019.

O processo de revisão se deu compreendendo uma vasta abordagem metodológica quanto a revisões de síntese, para que fosse possível a combinação de dados da literatura provenientes de estudos que empregaram diversas metodologias (SOUZA, SILVA e CARVALHO, 2010; FERREIRA et al., 2019).

O estudo de revisão integrativa foi elaborado baseando-se nas seis fases de construção propostas por Souza, Silva e Carvalho (2010): elaboração da pergunta norteadora, busca ou amostragem na literatura, coleta de dados, análise crítica dos estudos incluídos, discussão dos resultados e apresentação da revisão integrativa. $\mathrm{Na}$ etapa inicial, foi definida a pergunta central que orientou o estudo: "Quais são os impactos do processo de atenção farmacêutica para pacientes diagnosticados com doenças crônicas não transmissíveis?".

Posteriormente, na fase de busca ou amostragem na literatura, selecionaramse os termos- chaves, seguindo a orientação dos descritores em ciências da saúde (DeCS): "pharmaceutical care" or "pharmaceutical services" and "chronic diseases" and "diabetes" and "hypertension" no SciELO e no PubMed/MEDLINE. Ambos os sites foram acessados em novembro e dezembro de 2019. Para a seleção dos trabalhos, foram considerados os seguintes critérios de inclusão: artigos originais derivados de pesquisas; trabalhos que tratavam dos serviços farmacêuticos clínicos desenvolvidos na atenção primária no Brasil; trabalhos publicados nos idiomas português, inglês ou espanhol no período de 2004 a 2019. 
Excluíram-se os trabalhos duplicados, de revisão da literatura, sobre os serviços farmacêuticos clínicos desenvolvidos em universidades, institutos de pesquisa, hospitais, drogarias e farmácias privadas, nos Centros de Atenção Psicossocial (CAPSs) e em unidades básicas de saúde cujas atividades não contavam com a participação do farmacêutico.

A sistematização do recrutamento das publicações elegíveis obtidas nas bases consultadas está representada na forma de fluxograma (Figura 1), com a descrição do processo de busca e o respectivo quantitativo de estudos localizados em cada um dos sites. Além disso, para subsidiar a discussão, foram confrontados os dados desses artigos com os de outros estudos, oriundos de pesquisas nacionais e internacionais.

Figura 1. Fluxograma de seleção de artigos conforme critérios de inclusão e exclusão

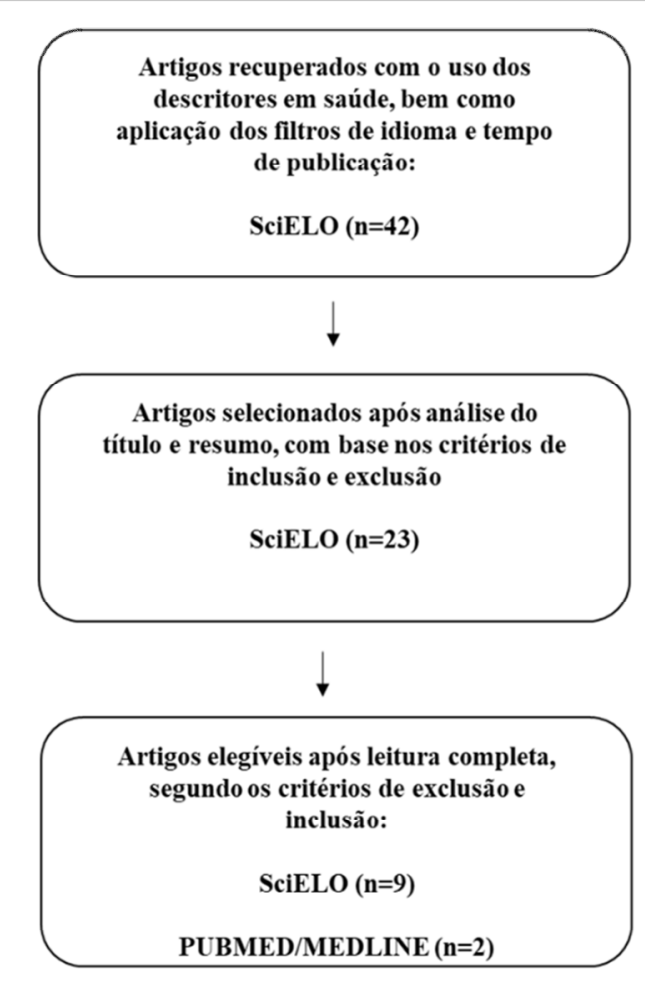

Fonte: de Sousa, et al. 2020 


\section{RESULTADOS E DISCUSSÃO}

A Atenção Farmacêutica (AF) é uma prática que tem como principal finalidade melhorar a qualidade de vida do paciente que faz uso de medicamentos, seja ele contínuo ou não. Dessa forma, otimizar o tratamento farmacológico e prevenir problemas relacionados ao uso de medicamentos são uns dos objetivos principais desse modelo de assistência à saúde (RENOVATO, 2004).

Através do acompanhamento farmacoterapêutico, o farmacêutico visa garantir o tratamento mais indicado, efetivo, seguro e conveniente a esses usuários. Logo, a orientação do seu trabalho é deslocada do produto para o serviço e do medicamento para o paciente, considerando-o na sua totalidade, valorizando a ocorrência de problemas de saúde relacionados a medicamentos e buscando resolvê-los através da intervenção farmacêutica no contexto da equipe interdisciplinar, com a aplicação de medidas preventivas ou corretivas ligadas diretamente ao uso racional de medicamentos (AIRES, 2010).

O processo de atenção farmacêutica voltado à pacientes portadores de doenças crônicas, visa melhorar a farmacoterapia, consequentemente a qualidade de vida dessas pessoas. Nesse universo, o farmacêutico tem papel importante na atenção, por exemplo, ao paciente diabético, uma vez que este necessita de uma mudança em seu estilo de vida, como cuidados específicos no armazenamento da insulina. Há casos em que os pacientes são analfabetos e não possuem um nível de conhecimento da doença. O farmacêutico então, pode traçar estratégias para que o paciente consiga fazer o tratamento sem interrupções e com segurança, levando a uma consequente redução na mobimortalidade. Assim, pode-se dizer que a adesão ocorre quando o usuário está suficientemente motivado a continuar com o tratamento prescrito devido à percepção dos benefícios e resultados positivos (BEZERRA, 2009).

Na última década, a OMS mostrou também que cerca de $80 \%$ dos óbitos por DCNT ocorreram em países de baixa ou média renda, com $29 \%$ dos óbitos em 
adultos com menos de 60 anos, enquanto naqueles de alta renda esse percentual era de apenas $13 \%$.

Apesar de nos últimos anos a prática de exercícios físicos e uma alimentação equilibrada terem sido amplamente discutidos, ainda assim, há esse aumento nos números de DCNT por fatores de risco modificáveis, como padrão de alimentação inadequada (alto consumo de sódio, alimentos ultra processados, ingestão de gorduras, açúcar, baixa ingestão de vegetais) e baixa adesão à prática de exercícios físicos, que são de extrema importância na prevenção dessas doenças assim como no tratamento dos portadores, além do tabagismo e consumo de álcool.

Nesse contexto, o diabetes mellitus (DM) é entendido como um distúrbio no qual a concentração sérica de glicose encontra-se anormalmente elevada, pois o organismo não libera ou não utiliza a glicose de modo adequado. Essa concentração sérica de açúcar varia durante o dia, aumentando após uma refeição e retornando ao normal em 2 horas. Normalmente, a glicose encontra-se entre 70 e $110 \mathrm{mg} / \mathrm{dl}$ de sangue pela manhã, após uma noite de jejum. Também se encontra inferior a 120 a $140 \mathrm{mg} / \mathrm{dl}, 2$ horas após o consumo de alimentos ou de líquidos que contenham açúcares ou outros carboidratos (BRUTTI, 2019). O paciente passa a ser diagnosticado como diabético quando a concentração de glicose no sangue ultrapassa 110 mg/dl (pela manhã e em jejum).

Adicionalmente, dentro da classificação das DCNT, a hipertensão arterial sistêmica (HAS) é uma condição clínica multifatorial caracterizada por elevação sustentada dos níveis pressóricos $\geq 140$ e/ou $90 \mathrm{mmHg}$. Frequentemente se associa a distúrbios metabólicos, alterações funcionais e/ou estruturais de órgãos-alvo, sendo agravada pela presença de outros fatores de risco, como dislipidemia, obesidade abdominal, intolerância à glicose e diabetes (MALACHIAS, 2016).

No Brasil, HAS atinge 32,5\% (36 milhões) de indivíduos adultos, mais de $60 \%$ dos idosos, contribuindo direta ou indiretamente para $50 \%$ das mortes por doença cardiovascular (DCV), inclusive a insuficiência cardíaca congestiva (ICC). Junto com o DM, suas complicações possuem um impacto elevado na perda da produtividade do trabalho e da renda familiar, estimada em US\$ 4,18 bilhões entre 2006 e 2015 (MALACHIAS, 2016). 
A possibilidade de associação da hipertensão arterial e do diabetes mellitus é da ordem de $50 \%$, o que, não raro, requer o manejo das duas doenças no mesmo usuário, agravado pelo fato de que sua concomitância potencializa o dano micro e macrovascular decorrente, acarretando alta morbidade cardiocerebrovascular. Além desses, a hipertensão arterial e o diabetes mellitus ainda apresentam outros aspectos em comum: etiopatogenia; fatores de risco; tratamento não medicamentoso; caráter crônico; previsibilidade; assintomáticos em estágios iniciais; difícil adesão ao tratamento; requisição de acompanhamento por equipe multidisciplinar e fácil diagnóstico (SANTOS, 2012).

Com o intuito de minimizar os danos decorrentes de tais afecções, o Ministério da Saúde implantou o Plano de Reorganização da Atenção à Hipertensão Arterial e ao Diabetes Mellitus e criou o Sistema de Cadastramento e Acompanhamento de Hipertensos e Diabéticos (HIPERDIA), utilizado em todos os Centros de Saúde da Família (CSF) (SANTOS, 2012).

Dadas às suas características crônicas e muitas vezes incapacitantes, a baixa adesão ao tratamento acarreta complicações de caráter emergencial e muitas vezes irreversíveis, afetando diretamente na qualidade de vida pessoal e familiar. Baixa adesão justificada pelos fatores condicionantes como o uso inapropriado de medicamentos decorrente de esquemas terapêuticos polifármacos, orientações a respeito da mudança de estilo de vida não condizentes com a realidade do indivíduo e nutrição empobrecida (LOPES, 2020).

No Brasil, a assistência farmacêutica engloba um conjunto de ações que são: a seleção, programação, aquisição, distribuição, dispensação, garantia de qualidade dos produtos e serviços, bem como a sua avaliação e acompanhamento de seu uso, com o objetivo de obter resultados que estejam diretamente ligados a melhoria da saúde da população (BRASIL, 2006). Segundo Starfield (2004), por definição, a Atenção Primária à Saúde se dá como uma estratégia de organização da atenção à saúde voltada para responder de forma regionalizada, contínua e sistematizada à maior parte das necessidades de saúde de uma população, integrando ações preventivas e curativas, bem como a atenção a indivíduos e comunidades.

A maior dificuldade que se enfrenta quando falamos de orientação voltada para o modelo de AF nas redes públicas de assistência à saúde de pacientes 
crônicos, é o profissional se fazer valorizado tanto pelos pacientes como pela equipe de trabalho. Infelizmente, ainda existe o estigma de que o farmacêutico é o profissional apenas da dispensação medicamento, por mais que isso não se aplique ao presente da profissão. O principal instrumento de trabalho farmacêutico são os fármacos, mas os profissionais farmacêuticos acabaram evoluindo o pensamento clínico para um entendimento que aproximasse as necessidades da população para redefinir diversos conceitos assistenciais (OLIVEIRA, et al. 2010).

Desta forma, a efetividade da atuação clínica do farmacêutico tem sido consistentemente demonstrada em estudos por meio da melhoria da qualidade de vida dos pacientes crônicos, da identificação e resolução de problemas relacionados aos medicamentos (PRM's), da adequação de parâmetros clínicos e da redução de gastos para doenças crônicas, no próprio sistema público (MAGID, et al. 2013). Portanto, isso evidencia que a inserção de serviços voltados para o cuidado farmacêutico na prestação de assistência à saúde em pacientes crônicos constitui estratégia eficaz para promover impactos clínicos, humanísticos e econômicos.

\section{CONCLUSÃO}

Quando levamos em consideração abordagens estratégicas para a construção de cenários de assistência à saúde, acabamos por promover ações diretamente relacionadas ao serviço de assistência farmacêutica. Especificamente nos casos de assistência a pacientes com alguma DCNT, a atenção farmacêutica precisa estar focada na equipe de cuidados básicos de serviços de saúde e nas populações-alvo. Os dados levantados por essa revisão integrativa, revelam a importância dos processos educacionais destinados à qualificação educacional dos farmacêuticos do sistema de saúde brasileiro, utilizando a perspectiva transdisciplinar para fornecer conhecimento sobre a realidade da situação, organização e gestão da saúde, voltadas para doenças como hipertensão e diabetes. 
A tomada de decisões clínicas compartilhada por equipes multidisciplinares, podem então, ser direcionadas para resolução de problemas de saúde crônicos. O presente estudo, também nos leva a refletir se a prática clínica empregada durante a implementação do tratamento farmacêutico é consistente com a prática clínica que o contexto exige e favorece. É possível que o processo de implementação da atenção farmacêutica para diferentes grupos de pacientes, tenha dificuldades devido a tentativas de replicar modelos teóricos que possam desconsiderar a realidade complexa dos sistemas de saúde, principalmente nos países em desenvolvimento. Dessa forma, mecanismos que busquem identificar e trabalhar com necessidades reais favorecem a geração e o compartilhamento de conhecimentos, o trabalho colaborativo, a reavaliação e o planejamento constante, facilitadores do processo de implantação dos serviços de atenção farmacêutica a nível nacional.

\section{REFERÊNCIAS BIBLIOGRÁFICAS}

AIRES, C.C.N.F.; MARCHIORATO L. Acompanhamento farmacoterapêutico a hipertensos e diabéticos na unidade de saúde Tereza Barbosa: análise de caso. R. Bras. Farm. Hosp. Serv. Saúde, São Paulo, v.1, n.1, p. 1-24, set./dez. 2010.

BEZERRA, D.S.; SILVA, A.S.; CARVALHO, A.L.M. Avaliação das características dos usuários com hipertensão arterial e/ou diabetes mellitus em uma Unidade de Saúde Pública, no município de Jaboatão dos Guararapes-PE, BRASIL. Rer. Ciênc. Farm. Básica Apl., 30(1), p.69-73, 2009.

BRASIL. Ministério da Saúde. Secretaria de Ciência, Tecnologia e Insumos Estratégicos. Assistência farmacêutica na atenção básica: instruções técnicas para sua organização. 2. ed. Brasília: Ministério da Saúde; 2006.

BRUTTI, B. et al. Diabete Mellitus: definição, diagnóstico, tratamento e mortalidade no Brasil, Rio Grande do Sul e Santa Maria, no período de 2010 a 2014. Braz. J. Hea. Rev., Curitiba, v. 2, n.4, p. 3174-3182, jul./ago. 2019.

DUNCAN, B. B. et al. Doenças crônicas não transmissíveis no Brasil: prioridade para enfrentamento e investigação. Rev. Saúde Pública, v.46, (Supl):126-134, 2012.

FAGUNDES, C.N.; CORSO, A.C.T.; CHICA, D.A.G. Perfil epidemiológico de hipertensos e diabéticos na atenção básica em saúde, Florianópolis- SC. Rev. Pesq. Saúde, v.18 (1), p. 2834, jan-abr, 2017.

FERREIRA, L. et al. Educação permanente em saúde na atenção primária: uma revisão integrativa da literatura. Saúde em Debate, Rio de Janeiro, v. 43, n. 120, p. 223-239, 2019.

GOUVEIA, W. A. At center stage: Pharmacy in the next century. Am. J. Health-Syst Pharm. v.56, p.2533, 1999. 
LOPES, J.R.; XAVIER, B.L.S.; PEREIRA, F.M.V. Perfil epidemiológico de usuários atendidos em ação de saúde na baixada litorânea do Rio de Janeiro. Rev Fun Care Online, v.12, p. 257262, jan/dez, 2020.

MAGID, D.J., OLSON, K.L., BILLUPS, S.J., et al. A pharmacist-led, American Heart Association Heart360 Web-enabled home blood pressure monitoring program. Circ Cardiovasc Qual Outcomes. 6(2):157-63, 2013.

MALACHIAS, M.V.B., et al. Diretriz Brasileira de Hipertensão Arterial: Capítulo 1 Conceituação, Epidemiologia e Prevenção Primária. Arq. Bras. Cardiol. v.107, n.3 supl.3 São Paulo Sept. 2016.

OLIVEIRA, L.C.F, ASSIS, M.M.A, BARBONI, A.R. Assistência Farmacêutica no Sistema Único de Saúde: da Política Nacional de Medicamentos à Atenção Básica à Saúde. Ciênc. Saúde coletiva. 2010.

RENOVATO, R.D.; TRINDADE, M.F. Atenção farmacêutica na hipertensão arterial em uma farmácia de Dourados, Mato Grosso do Sul. Rev. Infarma, v.16, n. 11-12, p. 49-55, 2004.

SANTOS, J. C.; MOREIRA T.M.M. Fatores de risco e complicações em hipertensos/diabéticos de uma regional sanitária do nordeste brasileiro. Rev. esc. enferm. USP vol.46 no.5 São Paulo Oct. 2012.

STARFIELD, B. Atenção Primária: equilíbrio entre necessidades de saúde, serviços e tecnologia. Brasília: Unesco Brasil/ Ministério da Saúde 2004.

SOUZA, M.T.; SILVA, M.D.; CARVALHO, R. Revisão integrativa: o que é e como fazer. Einstein, São Paulo, v. 8, n. 1, p. 102-106, 2010. 